\title{
Joint Planning of Natural Gas and Electric Power Transmission with Spatially Correlated Failures
}

\author{
Wenjing $\mathrm{Su}$ \\ Penn State University \\ wzs167@psu.edu
}

\section{Abstract}

We develop and illustrate a method for the joint planning of natural gas and electric power systems that are subject to spatially correlated failures of the kind that would be expected to occur in the case of extreme weather events. Our approach utilizes a two-stage stochastic planning and operations framework for a jointly planned and operated gas and electric power transmission system. Computational tractability is achieved through convex relaxations of the natural gas flow equations and the use of a machine learning algorithm to reduce the set of possible contingencies. We illustrate the method using a small test system used previously in the literature to evaluate computational performance of joint gas-grid models. We find that planning for geographically correlated failures rather than just random failures reduces the level of unserved energy relative to planning for random (spatially uncorrelated failures). Planning for geographically correlated failures, however, does not eliminate the susceptability of the joint gas-grid system to spatially uncorrelated failures.

\section{Nomenclature}

\section{Power System Sets}

$N^{e} \quad$ Set of electric power buses (nodes)

$\Omega \quad$ Set of power generators

$\Omega^{g f} \quad$ Set of gas-fired power generators, $\Omega^{g} \subseteq \Omega$

$\Omega^{n g f}$ Set of non-gas-fired power generators, $\Omega^{n g} \subseteq$ $\Omega$

$\Gamma_{i} \quad$ Set of generators connected to bus $i$

$N_{i}^{e} \quad$ Set of buses connected to bus $i$ by an edge

$A^{e} \quad$ Set of power transmission lines

\section{Power System Parameters}

$\delta_{0} \quad$ Index of the reference bus

$P_{i}^{l} \quad$ Nodal active power load at bus $i$

\author{
Seth Blumsack \\ Penn State University and Santa Fe Institute \\ blumsack@psu.edu
}

$\underline{P_{i}^{e}}, \overline{P_{i}^{e}}$ Active power generation limits of generator $i$

$C_{1}^{i}, C_{2}^{i}$ Cost coefficients of power generator $i$

$H_{i}$ Heat rate coefficient of gas-fired power generators

$X_{i j} \quad$ Reactance of a transmission line

$\overline{F_{i j}} \quad$ Thermal limit/capacity of a transmission line

$M \quad$ A large penalty constant for Big M method

\section{Power System Variables}

$p_{i j, k} \quad$ Active power of a transmission line in event $k$

$p_{j, k}^{e} \quad$ Active power output of generator $j$ in event $k$

$\theta_{i, k} \quad$ Phase angle at bus $i$ in event $k$

$u s e_{i, k} \quad$ Unserved electricity demand of node $i$ in event $k$

\section{Natural Gas System Sets}

$N^{g} \quad$ Set of natural gas junctions (nodes)

$A^{g} \quad$ Set of all links joining a pair of junctions

$T_{i} \quad$ Set of gas-fired power plants connected to node $i$

$A^{p} \quad$ Set of base pipelines, subset of $A^{g}$

$A^{c} \quad$ Set of base compressors, subset of $A^{g}$

\section{Natural Gas System Parameters}

$W_{a} \quad$ Pipeline resistance (Weymouth) factor

$\underline{P D_{i j}}, \overline{P D_{i j}}$ Pressure drop limits from junction $i$ to juntion $j$

$\underline{\pi_{i}}, \overline{\pi_{i}} \quad$ Squared pressure limits at junction $i$

$\alpha_{i j}^{c}, \overline{\alpha_{i j}^{c}}$ Compression limits squared at compressor station

$D_{i} \quad$ Firm gas consumption at junction $i$

$\underline{D_{i}}, \overline{D_{i}}$ Gas consumption limits at junction $i$

$\underline{S_{i}}, \overline{S_{i}} \quad$ Gas product limits at junction $i$

$Y_{i} \quad$ Cost coefficient of gas production at junction $i$

Natural Gas System Variables

$\pi_{i, k} \quad$ Squared pressure of gas node $i$ in event $k$

$x_{i j, k} \quad$ Gas flow on pipelines and compressors in event $k$

$\lambda_{i j, k} \quad$ Auxiliary relaxation variable in event $k$ 
$d_{i, k} \quad$ Gas consumption at junction $i$ in event $k$

$s_{i, k} \quad$ Gas production at junction $i$ in event $k$

$y_{i j, k}^{+}, y_{i j, k}^{-}$Binary flow direction for links in event $k$

Extreme Events and Expansion Sets

$k \in K$ Set of extreme weather and climate events

$\Lambda^{t} \quad$ Set of transmission expansion candidates

$\Lambda^{p} \quad$ Set of pipeline expansion candidates

$\Phi_{k}^{t} \quad$ Set of transmission lines impacted in event $k$

$\Phi_{k}^{p} \quad$ Set of pipelines impacted in event $k$

Extreme Events and Expansion Parameters

$\beta_{i j}^{p} \quad$ Expansion cost of a pipeline

$\beta_{i j}^{t} \quad$ Expansion cost of a transmission line

$\eta \quad$ Penalty cost for $1 \mathrm{MWh}$ of unserved energy

$S T_{i j, k}$ Binary status of an existing line in event $k$

$S P_{i j, k} \quad$ Binary status of existing pipelines in event $k$

Extreme Events and Expansion Variables

$u s e_{i, k} \quad$ Unserved electricity demand of node $i$ in event $k$

$u s g_{i, k} \quad$ Unserved gas demand of junction $i$ in event $k$

$z_{i j}^{p} \quad$ Binary expansion decision for pipeline candidates

$z_{i j}^{t} \quad$ Binary expansion decision for transmission line candidates

\section{Introduction}

Recent reports [1] [2] as well as experiences in Texas [3] and the U.S. Northeast [?] have shown that the U.S. energy system is vulnerable to extreme weather and climate events (EWCE). The increasing frequency of EWCE has led to public-sector actors at the federal level [4], [1], [5], state level [6] [?], [7] as well as utilities [8] to focus on planning critical energy infrastructure around such extreme events. Noting that planning for EWCE resilience requires different approaches than traditional power-sector generation or transmission planning, these actors have highlighted needs for vulnerability assessments including needs for vulnerability assessments and ways to identify optimal resilience measures.

Incorporating the risk of extreme weather and climate events into energy system planning process is a challenging task because EWCE are high-impact and low probability events; because the risk of such events is non-stationary in a changing climate [9]; and a single event can include multiple types of extreme weather (floods and landslides, for example) and multiple interconnected systems whose impacts compound on one another. This paper addresses a gap in the literatures on power system resilience and on models of coupled electricity and gas systems. We formulate and implement a joint planning and operations model for coupled gas-grid systems that is able to capture EWCE whose location and severity are uncertain and can result in spatially correlated outages that may not be captured in N-k types of planning models. Our approach utilizes a stochastic optimization formulation and a novel scenario-reduction approach based in machine learning.

\section{Literature Review}

The literature on planning for resilience has focused on quantifying vulnerability and enhancing resilience under low-probability and high-impact contingency scenarios for power systems. Some of literature does consider EWCE risk on the power system [10] [11][12]. Other power system models are planning models with operation stages where post-contingency dispatch model runs to determine the minimum possible impact during extreme events. The models vary greatly by the size [10][13] and components of power grids as well as the type of threats including natural disasters [14] and terrorism attacks[15][16]. For example, [17] proposed a distribution network planning model to minimize damage from hurricanes while [18] modeled transmission network under high wind events. To model the impact of natural hazards on power system, many studies have employed a similar framework: threat prediction, components exposure and vulnerability, system response, and system restoration [19]. Threats from natural hazards can be modeled as one type of hazard at a time, the most common approach, or as simultaneous weather and climate events. It is important to evaluate the joint probability and consequences when infrastructure systems are planned because catastrophic damage from compound extreme events is bigger than sum of individual extreme events[9]. [12] assessed the potential impact of windstorms on transmission lines and towers as well as the impact of floods on substations and power plants.

There are short-term resilience measures before, during or after the EWCE such as reserve planning and allocation of repair crews [18] and long-term resilience measures such as installing energy storage or new transmission lines [20]. Resilience measures can also be categorized as building new infrastructure or hardening existing infrastructure. These measures are evaluated by running operation models to compare their effectiveness[12] or by robust optimization or stochastic optimization methods when considering the uncertainty of EWCE. Sequential or non-sequential Monte Carlo (MC) algorithm techniques are employed 
to account for uncertainty of extreme weather and climate events [21]. Sequential MC is necessary if the model considers restoration processes such as including constraints of repairing crew number/resource or travel time to repair location [22]. Spatial or temporal components can be incorporated into the model indirectly by using historical or predictive geographic-specific extreme weather and climate events data [10]. With the uncertainty of EWCE included, resilience planning model is commonly formulated as a defender-attacker-defender game model[17] which is usually a two-stage robust optimization model. The defender plans system expansion based on the worst case scenario where the attacker causes the maximum damage. Among all uncertain scenarios, the defender redispatches the power system to reduce the damage as much as possible. The Column and Constraint Generation algorithm is usually used to solve the robust optimization problem [17]. For the problem that is formulated as a stochastic optimization model, [20] uses Optimization via Simulation method to solve and [23] constructed a heuristic method to find the solution strategy.

A gap in the literature on coupled gas-grid planning in particular that our approach seeks to fill is incorporating resiliency as a planning objective instead of solely minimizing total cost to meet energy demand. [24] designed a combined electricity and gas expansion model (CEGE) to satisfy coincident high demand scenarios and designed test system for the United States using publicly available data. The electricity system is integrated with natural gas system via the heat rate coefficients of gas-fired power plants. The CEGE model was then updated to add gas-price elasticity caused by congestion on existing models [25]. However, most existing models do not consider resilience as a constraint or an objective except the integrated model in [14][26]. [14] and [26] both build a tri-level two-stage robust optimization model for joint power and gas model. [14] assumes that the natural gas pipelines are robust to natural disasters and harden the power system by replacing transmission lines with underground gas pipelines. In [26], both power and gas system components are vulnerable to natural disasters, but the spatial correlation of outages resulted from natural disasters are not studied.

The remainder of the paper is organized as follows. Section III provides the mathematical formulation for the stochastic optimization problem while Section IV describes the experimental design of how to simulate the EWCE with the test system. Simulation results and conclusions are presented in section $\mathrm{V}$ and VI respectively.

\section{Model Formulation}

In this section, we provided the formulation for a two-stage stochastic optimization model of joint power and gas system. The equations for the operation stage will hold for all scenarios in the set of extreme weather and climate events $K$.

\subsection{Constraints for Gas System}

The gas flow balance is preserved at junctions by the following constraints.

$$
\begin{aligned}
s_{i, k} & -d_{i, k}+\sum_{(j, i) \in A^{g}} x_{j i, k}-\sum_{(i, j) \in A^{g}} x_{i j, k} \\
& +\sum_{(j, i) \in \Lambda^{p}} x_{j i, k}-\sum_{(i, j) \in \Lambda^{p}} x_{i j, k}=0 \quad \forall i \in N^{g}
\end{aligned}
$$

The binary flow direction variables need to be constrained for all pairs of links between the nodes including pipelines and compressors.

$$
\begin{array}{ll}
y_{i j, k}^{+}+y_{i j, k}^{-}=S P_{i j, k} & \forall(i, j) \in A^{p} \\
y_{i j, k}^{+}+y_{i j, k}^{-}=z_{i j}^{p} & \forall(i, j) \in \Lambda^{p} \\
y_{i j, k}^{+}+y_{i j, k}^{-}=1 & \forall(i, j) \in A^{c}
\end{array}
$$

The flow direction in pipelines should aligns with the sign of the flow. Equations (5) and (6) represent those constraints that apply for both existing pipelines and new pipelines. The pressure drop across pipelines are consistent with the sign of the flow as in equation (7) and (8) for both existing pipeline set and pipeline candidate set.

$$
\begin{aligned}
& -\left(1-y_{i j, k}^{+}\right) \sum_{i \in N^{g}} \overline{S_{i}} \leq x_{i j, k} \\
& x_{i j, k} \leq\left(1-y_{i j, k}^{-}\right) \sum_{i \in N^{g}} \\
& \left(1-y_{i j, k}^{+}\right)\left(\underline{\pi_{i}}-\overline{\pi_{i}}\right) \leq \pi_{i, k}-\pi_{j, k} \\
& \pi_{i, k}-\pi_{j, k} \leq\left(1-y_{i j, k}^{-}\right)\left(\underline{\pi_{i}}-\overline{\pi_{i}}\right)
\end{aligned}
$$

Weymouth equations make sure that the gas flow in a pipeline satisfy the physical constraints of fluid mechanics. Because the Weymouth equation is not convex, we use bilinear relaxations introduced by McCormick and the second-order cone relaxation which is often tight for expansion planning problem. The relaxation variable $\lambda_{i j, k}$ is in $\left\langle y_{i j, k}^{+}-y_{i j, k}^{-}, \pi_{i, k}-\right.$ $\left.\pi_{j, k}\right\rangle$, which are represented by equations (9)-(12). The 
relaxation applies to both existing pipelines and pipeline candidates.

$$
\begin{gathered}
\lambda_{i j, k} \geq \pi_{j, k}-\pi_{i, k}+\left(\underline{\pi_{i}}-\overline{\pi_{j}}\right)\left(y_{i j, k}^{+}-y_{i j, k}^{-}+1\right) \\
\lambda_{i j, k} \geq \pi_{i, k}-\pi_{j, k}+\left(\overline{\pi_{i}}-\underline{\pi_{j}}\right)\left(y_{i j, k}^{+}-y_{i j, k}^{-}-1\right) \\
\lambda_{i j, k} \leq \pi_{j, k}-\pi_{i, k}+\left(\overline{\pi_{i}}-\underline{\pi_{j}}\right)\left(y_{i j, k}^{+}-y_{i j, k}^{-}+1\right) \\
\lambda_{i j, k} \leq \pi_{i, k}-\pi_{j, k}+\left(\underline{\pi_{i}}-\overline{\pi_{j}}\right)\left(y_{i j, k}^{+}-y_{i j, k}^{-}-1\right) \\
S P_{i j, k} \lambda_{i j, k} \geq W_{i j} x_{i j, k}^{2} \\
z_{i j, k}^{p} \lambda_{i j, k} \geq W_{i j} x_{i j, k}^{2} \\
\forall(i, j) \in A_{p}
\end{gathered}
$$

Limits on natural gas compression are ensured by Eqs.(15)-(18).

$$
\begin{aligned}
& \pi_{j, k}-\overline{\alpha_{i j}^{c}} \pi_{i, k} \leq\left(1-y_{i j, k}^{+}\right) \overline{\pi_{j}} \\
& \underline{\pi_{i j}^{c}} \pi_{i, k}-\pi_{j, k} \leq\left(1-y_{i j, k}^{+}\right) \overline{\pi_{i}} \\
& \pi_{i, k}-\pi_{j, k} \leq\left(1-y_{i j, k}^{-}\right) \overline{\pi_{i}} \\
& \pi_{j, k}-\pi_{i, k} \leq\left(1-y_{i j, k}^{-}\right) \overline{\pi_{j}}
\end{aligned}
$$

Additional constrains below are supposed to ensure no gas flows through the pipeline if that candidate pipeline is not built.

$$
-z_{i j}^{p} \sum_{i \in N^{g}} \overline{S_{i}} \leq x_{i j, k} \leq z_{i j}^{p} \sum_{i \in N^{g}} \overline{S_{i}} \quad \forall(i, j) \in \Lambda^{p}
$$

Consumption, production and pressure should fall within the limits for each gas junction. For junctions with fixed gas consumption, the difference between fixed gas consumption and the amount of gas demand that is met is the quantity of unserved gas demand as in Eq.(20). For junctions with flexible gas consumption, which are junctions connected to gas-fired power plants, the variable gas consumption is between the nodal gas consumption bounds as in Eq.(21).

$$
\begin{aligned}
& \underline{D_{i}} \leq d_{i, k} \leq \overline{D_{i}} \\
& d_{i, k}+u s g_{i, k}=D_{i} \\
& \underline{\pi_{i}} \leq \pi_{i, k} \leq \overline{\pi_{i}} \\
& \underline{S_{i}} \leq s_{i, k} \leq \overline{S_{i}}
\end{aligned}
$$

\subsection{Constraints for Power System}

The power system is represented by a DC power flow model. Eq.(24) enforces that the total flow into a node equals the total flow out of the node for all power nodes. The electricity demand that is not satisfied is represented by the unserved electricity to balance the nodal flow. Eqs.(25) and (26), and Eqs.(27) and (28) define the power flow equations via the Big $\mathrm{M}$ method and we use $10^{6}$ as the large penalty constant.

$$
\sum_{j \in \Gamma_{i}} p_{j, k}^{e}=\sum_{(i, j) \in A^{e}} p_{i j, k}+P_{i}^{l}-u s e_{i, k}+\sum_{(i, j) \in \Lambda^{t}} p_{i j, k}
$$$$
-\left(1-S T_{i j, k}\right) M \leq p_{i j, k}-\frac{\theta_{i, k}-\theta_{j, k}}{X_{i j}}
$$

$p_{i j, k}-\frac{\theta_{i, k}-\theta_{j, k}}{X_{i j}} \leq\left(1-S T_{i j, k}\right) M$

$-\left(1-z_{i j}^{t}\right) M \leq p_{i j, k}-\frac{\theta_{i, k}-\theta_{j, k}}{X_{i j}}$

$p_{i j, k}-\frac{\theta_{i, k}-\theta_{j, k}}{X_{i j}} \leq\left(1-z_{i j}^{2}\right) M$

Eqs. (29)-(32) describe the bounds on the power system variables. Eq.(30) ensures that no power flows through a transmission line if the transmission line is down during the extreme event $k$. Eq.(31) ensures that there is no electricity going through a new transmission line if it is not being built. Eq.(33) sets the phase angle of a slack bus to be 0 as a reference.

$$
\begin{array}{cc}
\frac{P_{i}^{e}}{} \leq p_{i, k}^{e} \leq \overline{P_{i}^{e}} & \forall i \in \Omega \\
-S T_{i j, k} V_{i j} \leq p_{i j, k} \leq S T_{i j, k} V_{i j} & \forall(i, j) \in A^{e} \\
-z_{i j}^{t} S_{i j} \leq p_{i j, k} \leq z_{i j}^{t} S_{i j} & \forall(i, j) \in \Lambda^{t} \\
0 \leq u s e_{i, k} \leq P_{i}^{l} & \forall i \in N^{e} \\
\theta_{\delta_{0}, k}=0 & \forall k \in K
\end{array}
$$

\subsection{Coupling Constraint between Power System and Gas System}

The gas system and electricity system are connected because gas system supplies fuel to gas-fired power plants. This is reflected by the constraint of power output and gas demand of power plants. The equality results in non-convexity. Therefore, we relax the equality condition of gas consumption by power plants 
into an inequality.

$$
d_{i, k}=\sum_{j \in \Gamma_{i}} H_{j} \times p_{j, k}^{e} \quad i \in N^{g}
$$

\subsection{Extreme Weather and Climate Events}

The binary status variables represent whether the components fail during an extreme weather and climate event. The status of energy system components is determined by the location of system component and event impacted region. If the component is within the region impacted by EWCE, the pipelines or transmission lines fail and will not have power or gas flow going through. Otherwise, the status parameter equals one representing that the component fully functions. We assume that the pipeline and transmission line candidates are designed to be robust and resilient to both geographically correlated failures and uncorrelated failures. They will always function in the operation stage if they are chosen to be built in the first stage.

$$
\begin{array}{ll}
S T_{i j, k}=0 & \text { if } \forall(i, j) \in \phi_{k}^{t}, \forall k \in K \\
S P_{i j, k}=0 & \text { if } \forall(i, j) \in \phi_{k}^{p}, \forall k \in K
\end{array}
$$

\subsection{Objective Function}

Objective function for the stochastic optimization model is stated in Eq.(37). It composes the expected operating cost and penalty cost for unserved energy of all possible scenarios in the operation stage and the expansion cost in the investment stage.

$$
\begin{aligned}
\min & \sum_{k \in K}\left[\sum_{i \in \Omega^{n g f}}\left(C_{1}^{i} p_{i, k}^{e}+C_{2}^{i} p_{i, k}^{e}\right)+\sum_{i \in N^{g}} Y_{i} s_{i, k}\right. \\
& \left.+\sum_{i \in N^{e}} 24 \eta \times u s e_{i, k}+\sum_{i \in N^{g}} 86400 \eta \times u s g_{i, k}\right] \\
& +\sum_{(i, j) \in \Lambda^{t}} \beta_{i j}^{t} z_{i j}^{t}+\sum_{(i, j) \in \Lambda^{p}} \beta_{i j}^{p} z_{i j^{p}}
\end{aligned}
$$

\section{4. experimental design/ test cases}

The test system we use was developed in [27]. The power network is the IEEE 14 bus test system and the gas network is a representation of Belgium natural gas system[28]. The size of the test system is small with 14 electric buses and 20 natural gas junctions. The locations of the power and gas network is shown in Figure 1. The electric buses that have gas-fired power plants and the gas junctions that supply gas to power system are circled in the Figure 1. Because of their geographic proximity, the transportation of gas from gas junction to gas-fired power plants are assumed to be functioning even if the extreme weather and climate events hit the area. In other words, as long as the gas junctions next to the electricity nodes have gas supply, the gas will have access to gas-fired power plants.

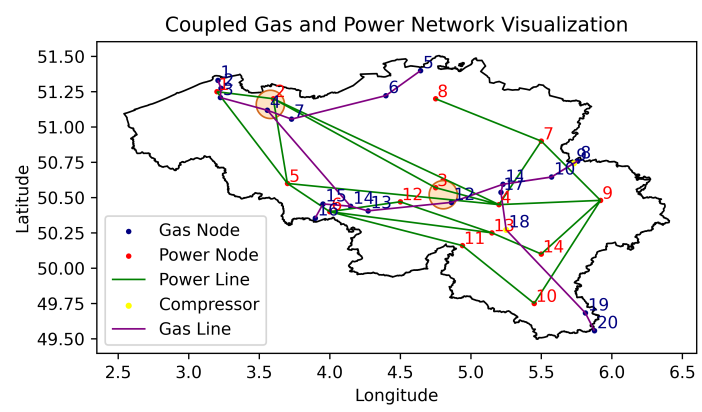

Figure 1. Joint Power and Gas System

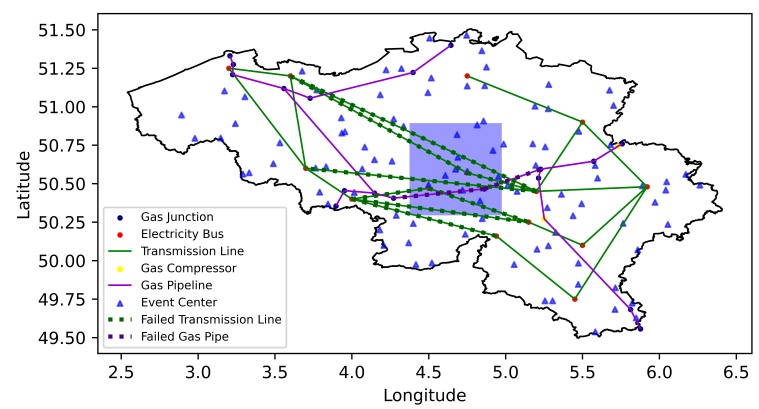

Figure 2. 100 Extreme Weather and Climate Event Centers with One Event as Example

The fundamental contingency feature that we consider in this paper is the spatial correlation of the location of the energy system components that are impacted in the contingency events. The extreme weather and climate events induce spatially correlated contingency events while most existing N-k contingency planning assumes the uncorrelatedness in the geographical distribution of the component failures. Therefore, we use geographically correlated failures(GCF) and geographically uncorrelated failures(UCF) to represent the extreme weather and climate events and contingency events in traditional N-k planning method respectively. Specifically, one EWCE is represented by an area/square area within where transmission lines and pipelines are affected. Multiple EWCEs that intersect with the scope of the 
test system are generated randomly using Monte Carlo to represent the uncertainty of climate risk. During each Monte Carlo run, a random event center with coordinate information is generated within the border of Belgium. A square box of a uniform size centering on the event center is defined as the extreme weather and climate event area. Transmission lines and pipelines intersecting the event area fail and stop functioning. The 100 extreme weather and climate event centers that we generated are presented in Figure 2.

To research the difference between two types of failure modes GCF and UCF, a set of equivalent contingency scenarios with geographically uncorrelated failures that have the same distribution of number of failed components are generated. The distribution of number of failed components is shown in Figure 3. Based on the number of failed transmission line and pipelines in GCF, same number of transmission lines and pipelines are randomly selected to be the failed components in the equivalent scenario with UCF. Therefore, there is no spatial correlation between failed components. We compare both the impact of two types of failure modes before the expansion and the investment decisions from the stochastic optimization model planning against the two types of contingency scenarios. To compare the joint system resilience against the two type of disasters, we run an one-stage operation model with base network structure under 100 GCF events and 100 UCF events. The metrics we used for comparison include the total cost or total unserved energy as well as the spatial distribution of unserved energy across gas consumer nodes and electricity demand nodes. We also look into the allocation of total unserved energy between two subsystems and the resilience of individual subsystem against two types of contingencies.

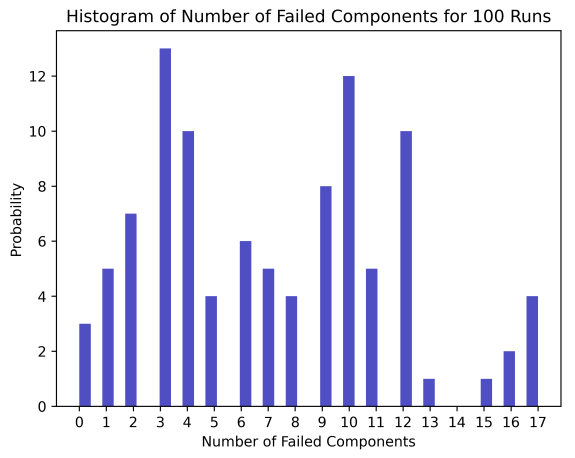

Figure 3. Distribution of Number of Failed Components for 100 Contingency Scenarios

The expansion model is a two-stage deterministic equivalent stochastic optimization model. The first stage is the planning stage to determine the investment decisions of natural gas pipelines and transmission lines while the second stage is an operation stage to redispatch the electricity and gas systems to have the minimal loss of load in each contingency scenario. The scenarios in the second stage are selected by the Approximate Latent Factor Algorithm (ALFA) from the set of $100 \mathrm{UCF}$ and the set of $100 \mathrm{GCF}$ scenarios that are generated by Monte Carlo. The scenario reduction method is needed because the uncertainty of EWCE locations for GCF and the possible combinations of failed components for UCF makes it critical to incorporate several scenarios while including many scenarios results in the heavy computational burden because of the non-linearity of the gas system and joint operation of two subsystems.ALFA is a method rooted in multivariate statistics, and it provides a set of scenarios and weights that can be incorporated into planning formulations[29]. After solving the stochastic optimization mode, the resilience of the expanded network based on the planning model against GCF and the planning model against UCF will be tested against the same set of 100 GCFs and 100 UCFs.

The flow diagrams in 4 and 5 summarize the steps of impact comparison and comparison of network expansion decisions. A detailed description about the flow diagrams are also written as the numbered lists below.

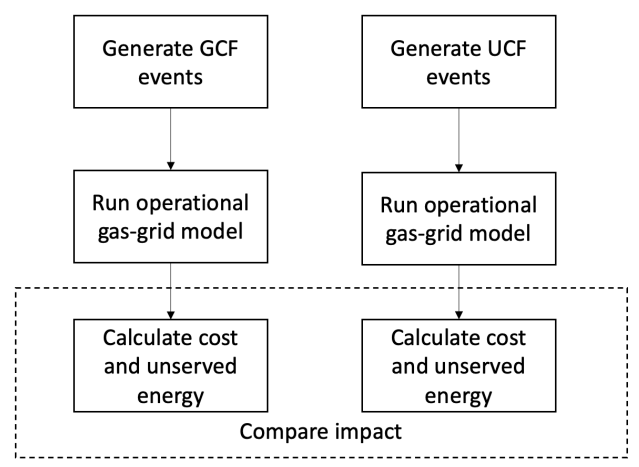

Figure 4. Flow diagram of impact comparison

\subsection{Comparison of Impact from Component Failures}

1. Generate 100 extreme weather and climate event areas within the model scope using Monte Carlo.

2. Run the operation model under 100 contingency scenarios with geographically correlated failures for base network without network expansion. 
3. Generate 100 equivalent contingency scenarios of uncorrelated failures with the same distribution of number of failed components.

4. Run the operation model under 100 scenarios under 100 uncorrelated failures for the base network without network additions.

5. Compare the impact of GCF and UCF on system using metrics including: total unserved energy, total system cost and average electricity price, nodal unserved energy and Gini coefficients of unserved energy as a way to measure spatial distribution.

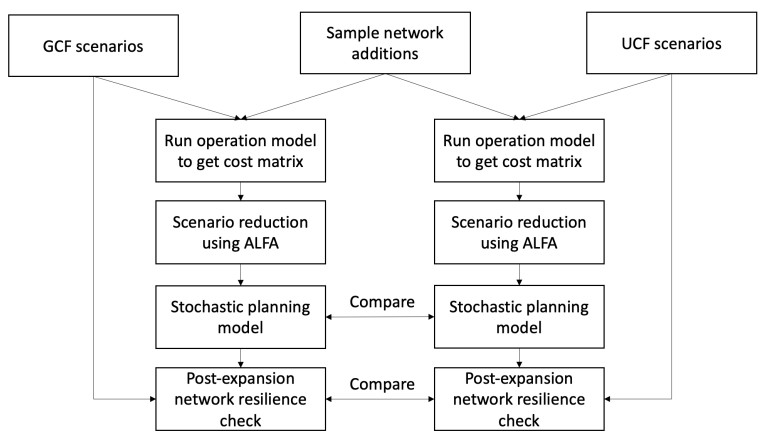

Figure 5. Flow diagram of expansion comparison

\subsection{Comparison of Stochastic Network Hardening Decisions}

1. Sample network expansion decisions as potential future network structures.

2. Run the operation model to obtain the two impact matrices with total cost as matrix entries for contingency scenarios with geographically correlated failures and uncorrelated failures for sample network structures.

3. Use Approximate Latent Factor Algorithm to select the representative contingency scenarios based on the cost matrix.

4. Run the deterministic equivalent for MINLP model under GCF and UCF with reduced contingency scenarios to get the expansion decisions.

5. Run the operation model under the GCF and UCF contingency scenarios with the two expanded network based on GCF planning model and UCF planning model to calculate the unserved energy.
6. Compare the expansion decisions from GCF and UCF planning models.

7. Run the operation model with the two expanded network structure to compare the resilience enhanced especially the amount of unserved energy reduced through network expansion.

\section{Numerical Results}

As described in the previous section, the experimental design is composed of impact comparison of joint system under geographically correlated failures and uncorrelated failures and comparison of planning model results. The results from impact comparison shows the vulnerability of existing network structure and proves the necessity of system planning processing considering extreme weather and climate events.

\subsection{Comparison of Impact Results}

Figure 6 captures the scenario-wise unserved electricity and gas demand from each contingency scenario. The unserved electricity demand accounts for a high percentage of total unserved energy demand in scenarios with GCF. Among contingency scenarios with $\mathrm{UCF}$, the unserved electricity demand is relatively low even when the system has high total unserved energy demand. This verifies that the power system is designed to be robust to spatially uncorrelated failures but not to correlated failures. The unserved gas demand accounts for a high percentage of total unserved energy under UCF, which can be explained by the high number of gas junctions with unserved gas demand.

To evaluate the spatial distribution of unserved energy across nodes, we use Gini coefficients as a measure. The formulation we use is im Eq (38). The higher the Gini coefficient, the greater the spatial concentration of unserved energy.

$$
G=\frac{\sum_{i=1}^{n}(2 i-n-1) x_{i}}{n \sum_{i=1}^{n} x_{i}}
$$

The cumulative distributions of Gini coefficients for gas demand nodes and electricity demand nodes under GCF and UCF are presented in Figure 7. First, regardless of the failure mode, the concentration of the unserved energy demand is high, which is a result of the small scale of the test system. Second, contingency scenarios with geographically correlated failures are more likely to have higher Gini coefficients as compared to uncorrelated failures. Third, the power system is more likely to yield a high Gini coefficient, which is 

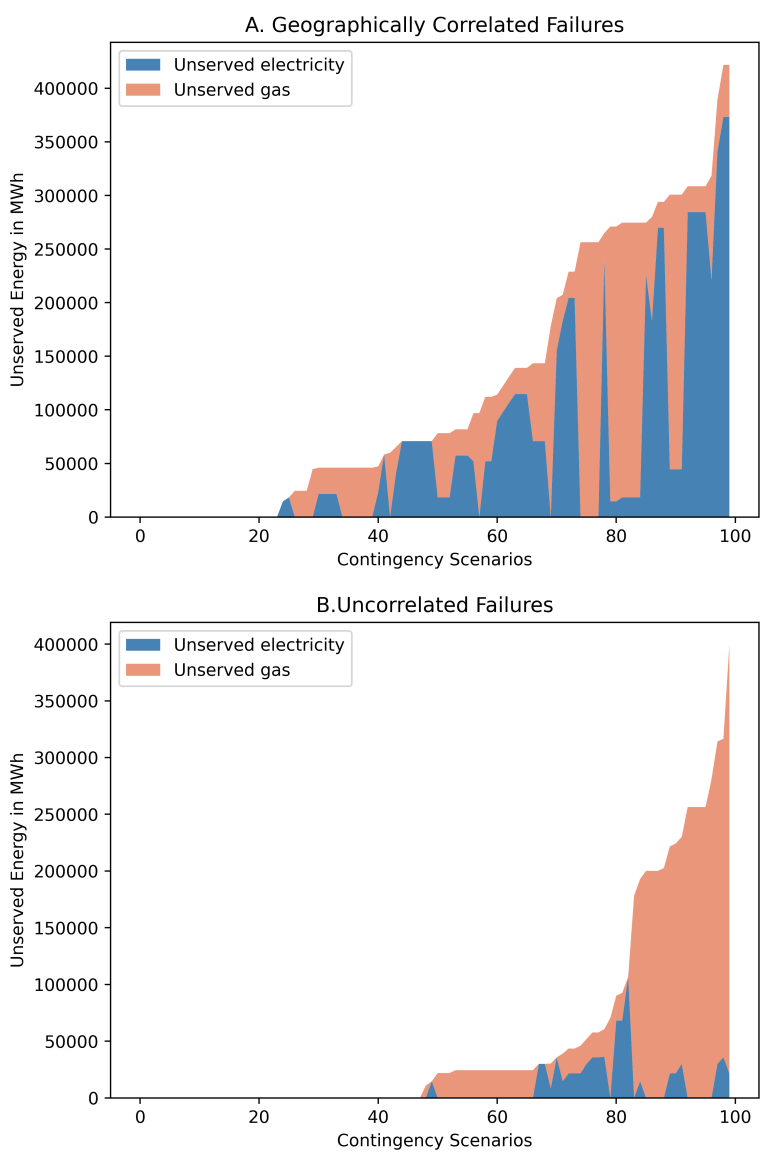

Figure 6. Stackplot of Unserved Energy

consistent with the redundancies built into the power network structure. Because an electricity demand node is less likely to be cut off from the grid entirely, the probability of having multiple electricity demand nodes being cut off is low. The unserved electricity demand is more likely to be concentrated in fewer number of nodes, which results in a high Gini coefficient.

\subsection{Comparison of Expansion Results}

For the same base network, the two-stage stochastic optimization model is run to obtain the expansion decisions on pipeline and transmission candidates. Because the test system is small, we assume an uniform price for all gas pipeline candidates and another price for all transmission line candidates. The MINLP model is solved with BARON solver. The planning model takes less than 2 hours to reach a solution, and unserved energy is driven to zero for all solutions.

The set of pipeline candidates and transmission line candidates is the same as the set of existing pipelines and transmission lines for the test system. The difference is

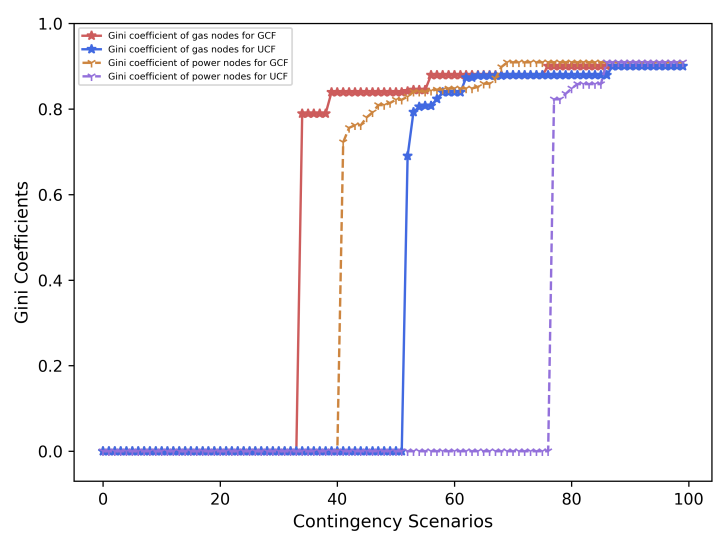

Figure 7. Gini Coefficients Comparison

that the sets of candidates are robust enough to operate normally in contingency scenarios with GCF or UCF. The locations of these candidates chosen are presented in 8 . There are five transmission lines being built from the planning model with GCF scenarios with uncertainty while there are only two transmission lines being built from that with UCF scenarios with uncertainty. Even though there are more lines being built in the GCF planning model, the set of two candidates chosen in the UCF planning model is not a subset of five candidates chosen in the GCF planning model. Thus,= planning for GCF does more than simply add extra redundancy as compared to planning for UCF. Seven pipelines are built from planning model with GCF or UCF scenarios. There is overlap between the new pipelines built, but the set of candidates chosen to be built is not identical in the two planning approaches.

After the system expansion, we test if the expanded network based on GCF or UCF planning is resilient to the two types of failures. We simulate operations of each of the post-expansion networks to the sets of GCF and UCF failures. Table 1 and Table 2 present the magnitude and and proportion of reduction of total unserved energy after system expansion based on GCF and UCF. Table 1 presents the reduction in unserved energy for a GCF-planned system that is subject to both GCF and UCF contingencies post-expansion. Table 2 presents the same information for a UCF-planned system. The Contingency Type column refers to whether the post-expansion system is subjected to GCF or UCF contingencies.

The expanded system based on planning considering contingency uncertainty with GCF reduces unserved energy effectively when facing geographically correlated failures. Unserved energy is reduced by $100 \%$ after expansion (i.e., post-expansion unserved energy is zero even in the face of contingencies) for 

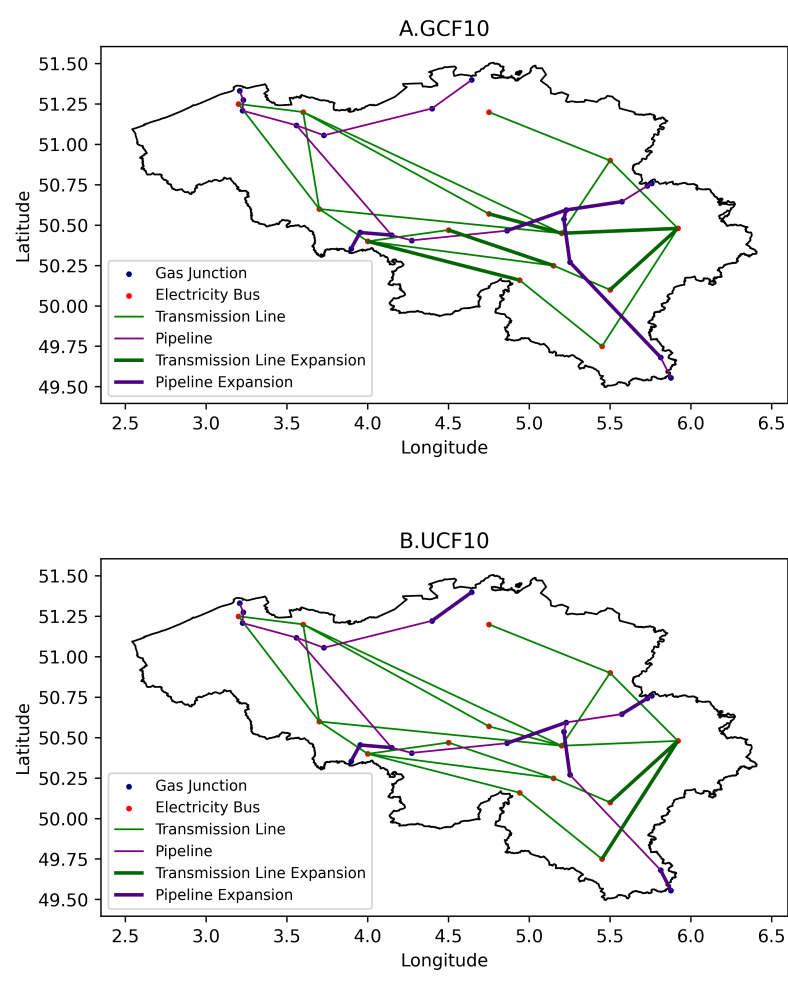

Figure 8. Locations of Expansion Decisions When Planning for GCF (Top) and UCF (Bottom)

more than $50 \%$ of the scenarios with unserved energy facing GCF. Planning against UCF is able to enhance the network robustness but cannot reduce the unserved energy by $100 \%$ when the system faces GCF. On the other hand, when the expanded network faces UCF, the planning considering GCF or UCF has a comparable performance. A reasonable explanation is that same number of pipelines are being built and more transmission lines are being built based on planning model considering GCF. Therefore, planning considering GCF does reduce unserved energy when facing UCF, but it is not cost-effective comparing to planning considering UCF. With the same budget, the planning model only considering GCF might have a worse performance in reducing unserved energy demand under UCF.

Table 1. Resilience of Expanded System Under Planning for GCF

\begin{tabular}{lccccc}
\hline Contingency Type & Min & $25 \%$ & $50 \%$ & $75 \%$ & Max \\
GCF in GWh & 0.0 & 11.6 & 116.0 & 256.4 & 421.8 \\
GCF in \% & $0 \%$ & $37.8 \%$ & $100 \%$ & $100 \%$ & $100 \%$ \\
UCF in GWh & 0.0 & 22.0 & 27.2 & 178.2 & 316.6 \\
UCF in \% & $0 \%$ & $48.5 \%$ & $89.7 \%$ & $100 \%$ & $100 \%$ \\
\hline
\end{tabular}

Table 2. Resilience of Expanded System Under Planning for UCF

\begin{tabular}{lccccc}
\hline \hline Contingency Type & Min & $25 \%$ & $50 \%$ & $75 \%$ & Max \\
GCF in GWh & 0.0 & 0.0 & 57.4 & 250.3 & 256.4 \\
GCF in $\%$ & $0 \%$ & $0 \%$ & $60.3 \%$ & $94.5 \%$ & $100 \%$ \\
UCF in GWh & 0.0 & 21.6 & 39.6 & 200.1 & 399.2 \\
UCF in $\%$ & $0 \%$ & $69.6 \%$ & $100 \%$ & $100 \%$ & $100 \%$ \\
\hline
\end{tabular}

\section{Conclusions}

The joint power and gas system shows vulnerability to both geographically correlated failures and uncorrelated failures. It is important to consider the low-probability and high-impact events in the system planning process. The power system is relatively resilient to uncorrelated failures comparing to geographically correlated failures because the N-k contingency analysis has been used in the planning process. The gas system shows vulnerability to both geographically correlated and uncorrelated failures. The difference in power and gas system robustness can be explained by their network topology. The mesh structure of the power system with high degree of connectivity is more resistant to contingency scenarios than the tree topology of the gas system. But the fuel dependency of power system on natural gas system is implicitly included in the model, which explains the vulnerability of power system in the results.

Network expansion based on planning for uncorrelated failures yields vulnerabilities when failtures are geographically correlated. Network expansion based on planning for correlated failures reduces unserved energy considerably, and in particular mitigates the scenarios with the highest levels of unserved energy demand. Network expansion based on planning for GCF or UCF exhibit comparable performance against uncorrelated failures. In part this result is due to the large number of transmission lines built under GCF planning. In other words, GCF planning is effective at mitigating spatially uncorrelated failures, but is not the most cost-effective way to mitigate those risks. Simply planning for one type of failure does not guarantee a robust energy system. It is crucial to consider the geographically correlated failures incuded by extreme weatehr and climate events besides the uncorrelated failure in the planning process for energy sector regulators and operators to achieve a resilient energy system.

\section{References}

[1] DOE, "Climate change and the US energy sector: Regional vulnerabilities and resilience solutions," tech. rep., October 2015.

[2] P. Hoffman and W. Bryan, "Comparing the impacts of 
northeast hurricanes on energy infrastructure," tech. rep., 2013.

[3] Electric Reliability Council of Texas, "Review of February 2021 extreme cold weather event." Accessed on April. 29 2021. [Online]. Available: http://www. ercot.com/content/wcm/key_ documents_lists/225373/2.2_REVISED_ ERCOT_Presentation.pdf, 2021.

[4] C. Zamuda, B. Mignone, D. Bilello, K. Hallett, C. Lee, J. Macknick, R. Newmark, and D. Steinberg, "US energy sector vulnerabilities to climate change and extreme weather," tech. rep., Department of Energy Washington DC, 2013.

[5] EPSA,DOE, "Climate change and the electricity sector guide for assessing vulnerabilities and developing resilience solutions to sea level rise," tech. rep., Department of Energy Washington DC, 2016.

[6] D. N. Bresch, E. W. Group, et al., "Building a resilient energy gulf coast: Executive report," tech. rep., ETH Zurich, 2010.

[7] E. Stein and A. Peltz, "Final order from NYPSC on Con Edison rate case reveals especially encouraging language on climate change directives." Accessed on April. 30 2021. [Online]. Available: http://blogs . edf.org/energyexchange/, 2014.

[8] Edison Electric Institute, "Before and after the storm update. a compilation of recent studies, programs, and policies related to storm hardening and resiliency," tech. rep., 2014.

[9] D. J. Wuebbles, D. W. Fahey, K. A. Hibbard, J. R. Arnold, B. DeAngelo, S. Doherty, D. R. Easterling, J. Edmonds, T. Edmonds, T. Hall, et al., "Climate science special report: Fourth national climate assessment (nca4), volume i," 2017.

[10] Y. Yang, W. Tang, Y. Liu, Y. Xin, and Q. Wu, "Quantitative resilience assessment for power transmission systems under typhoon weather," IEEE Access, vol. 6, pp. 40747-40756, 2018.

[11] R. Rocchetta, Y. Li, and E. Zio, "Risk assessment and risk-cost optimization of distributed power generation systems considering extreme weather conditions," Reliability Engineering \& System Safety, vol. 136, pp. 47-61, 2015.

[12] S. Espinoza, M. Panteli, P. Mancarella, and H. Rudnick, "Multi-phase assessment and adaptation of power systems resilience to natural hazards," Electric Power Systems Research, vol. 136, pp. 352-361, 2016.

[13] A. Bernstein, D. Bienstock, D. Hay, M. Uzunoglu, and G. Zussman, "Power grid vulnerability to geographically correlated failures - analysis and control implications," in IEEE INFOCOM 2014-IEEE Conference on Computer Communications, pp. 2634-2642, IEEE, 2014.

[14] C. Shao, M. Shahidehpour, X. Wang, X. Wang, and B. Wang, "Integrated planning of electricity and natural gas transportation systems for enhancing the power grid resilience," IEEE Transactions on Power Systems, vol. 32, no. 6, pp. 4418-4429, 2017.

[15] J. Salmeron, K. Wood, and R. Baldick, "Analysis of electric grid security under terrorist threat," IEEE Transactions on power systems, vol. 19, no. 2, pp. 905-912, 2004.
[16] A. L. Motto, J. M. Arroyo, and F. D. Galiana, "A mixed-integer lp procedure for the analysis of electric grid security under disruptive threat," IEEE Transactions on Power Systems, vol. 20, no. 3, pp. 1357-1365, 2005.

[17] W. Yuan, J. Wang, F. Qiu, C. Chen, C. Kang, and B. Zeng, "Robust optimization-based resilient distribution network planning against natural disasters," IEEE Transactions on Smart Grid, vol. 7, no. 6, pp. 2817-2826, 2016.

[18] M. Panteli, C. Pickering, S. Wilkinson, R. Dawson, and P. Mancarella, "Power system resilience to extreme weather: fragility modeling, probabilistic impact assessment, and adaptation measures," IEEE Transactions on Power Systems, vol. 32, no. 5, pp. 3747-3757, 2016.

[19] M. Panteli and P. Mancarella, "Influence of extreme weather and climate change on the resilience of power systems: Impacts and possible mitigation strategies," Electric Power Systems Research, vol. 127, pp. 259-270, 2015.

[20] T. Lagos, R. Moreno, A. N. Espinosa, M. Panteli, R. Sacaan, F. Ordonez, H. Rudnick, and P. Mancarella, "Identifying optimal portfolios of resilient network investments against natural hazards, with applications to earthquakes," IEEE Transactions on Power Systems, vol. 35, no. 2, pp. 1411-1421, 2019.

[21] R. Billinton and P. Wang, "Teaching distribution system reliability evaluation using monte carlo simulation," IEEE Transactions on Power Systems, vol. 14, no. 2, pp. 397-403, 1999.

[22] A. F. Mensah and L. Dueñas-Osorio, "Efficient resilience assessment framework for electric power systems affected by hurricane events," Journal of Structural Engineering, vol. 142, no. 8, p. C4015013, 2016.

[23] N. R. Romero, L. K. Nozick, I. D. Dobson, N. Xu, and D. A. Jones, "Transmission and generation expansion to mitigate seismic risk," IEEE Transactions on Power Systems, vol. 28, no. 4, pp. 3692-3701, 2013.

[24] C. B. Sanchez, R. Bent, S. Backhaus, S. Blumsack, H. Hijazi, and P. Van Hentenryck, "Convex optimization for joint expansion planning of natural gas and power systems," in 2016 49th Hawaii International Conference on System Sciences (HICSS), pp. 2536-2545, IEEE, 2016.

[25] R. Bent, S. Blumsack, P. Van Hentenryck, C. Borraz-Sánchez, and M. Shahriari, "Joint electricity and natural gas transmission planning with endogenous market feedbacks," IEEE Transactions on Power Systems, vol. 33, no. 6, pp. 6397-6409, 2018.

[26] M. Yan, Y. He, M. Shahidehpour, X. Ai, Z. Li, and J. Wen, "Coordinated regional-district operation of integrated energy systems for resilience enhancement in natural disasters," IEEE Transactions on Smart Grid, vol. 10, no. 5, pp. 4881-4892, 2018.

[27] C. Borraz-Sánchez, R. Bent, S. Backhaus, H. Hijazi, and P. V. Hentenryck, "Convex relaxations for gas expansion planning," INFORMS Journal on Computing, vol. 28, no. 4, pp. 645-656, 2016.

[28] D. De Wolf and Y. Smeers, "The gas transmission problem solved by an extension of the simplex algorithm," Management Science, vol. 46, no. 11, pp. 1454-1465, 2000.

[29] J. P. Bukenberger and M. D. Webster, "Approximate latent factor algorithm for scenario selection and 
weighting in transmission expansion planning," IEEE

Transactions on Power Systems, vol. 35, no. 2, pp. 1099-1108, 2019. 